\title{
Engineering Purely Nonlinear Coupling between Superconducting Qubits Using a Quarton
}

\author{
Yufeng Ye๑, ${ }^{1,2}$ Kaidong Peng $\odot,{ }^{1,2}$ Mahdi Naghiloo, ${ }^{2}$ Gregory Cunningham, ${ }^{2,3}$ and Kevin P. O’Brien $\oplus^{1,2, *}$ \\ ${ }^{1}$ Department of Electrical Engineering and Computer Science, Massachusetts Institute of Technology, \\ Cambridge, Massachusetts 02139, USA \\ ${ }^{2}$ Research Laboratory of Electronics, Massachusetts Institute of Technology, Cambridge, Massachusetts 02139, USA \\ ${ }^{3}$ Harvard John A. Paulson School of Engineering and Applied Sciences, Harvard University, \\ Cambridge, Massachusetts 02138, USA
}

(Received 1 December 2020; accepted 17 May 2021; published 29 July 2021)

\begin{abstract}
Strong nonlinear coupling of superconducting qubits and/or photons is a critical building block for quantum information processing. Because of the perturbative nature of the Josephson nonlinearity, linear coupling is often used in the dispersive regime to approximate nonlinear coupling. However, this dispersive coupling is weak and the underlying linear coupling mixes the local modes, which, for example, distributes unwanted self-Kerr nonlinearity to photon modes. Here, we use the quarton to yield purely nonlinear coupling between two linearly decoupled transmon qubits. The quarton's zero $\phi^{2}$ potential enables an ultrastrong gigahertz-level cross-Kerr coupling, which is an order of magnitude stronger compared to existing schemes, and the quarton's positive $\phi^{4}$ potential can cancel the negative self-Kerr nonlinearity of qubits to linearize them into resonators. This ultrastrong cross-Kerr coupling between bare modes of qubitqubit, qubit-photon, and even photon-photon is ideal for applications such as single microwave photon detection, ultrafast two-qubit gates, and readout.
\end{abstract}

DOI: 10.1103/PhysRevLett.127.050502

Introduction.-Circuit quantum electrodynamics (cQED) uses strong light-matter coupling in microwave superconducting circuits for quantum information processing [1,2]. In the ultrastrong regime [3], linear or field-field [4] coupling $g\left(\hat{a}-\hat{a}^{\dagger}\right)\left(\hat{\sigma}^{-}-\hat{\sigma}^{+}\right)$between artificial atoms and resonator photons can be of the same order of magnitude as the frequency of the modes $(g \sim 2 \pi \times 1 \mathrm{GHz} \gtrsim 0.1 \omega)$. However, to implement essential quantum operations such as qubit readout [4], gates $[5,6]$, single photon detection $[7,8]$, and bosonic qubit control [9], nonlinear or energy-energy [4] coupling $\chi \hat{a}^{\dagger} \hat{a} \hat{\sigma}_{z}$ is often required. Unfortunately, nonlinear coupling in cQED is much weaker than linear coupling $(\chi \ll g)$, which limits the speed of many nonlinear coupling dependent operations.

Nonlinear coupling is typically realized in the dispersive regime, where the linear coupling $g$ is chosen to be small vs the detuning: $g \ll \Delta=\left|\omega_{a}-\omega_{b}\right|$, so the Jaynes-Cummings Hamiltonian can be approximated $(\hbar=1$ hereafter),

Published by the American Physical Society under the terms of the Creative Commons Attribution 4.0 International license. Further distribution of this work must maintain attribution to the author(s) and the published article's title, journal citation, and DOI.

$$
\begin{aligned}
H_{\mathrm{JC}} & =\omega_{a} \hat{a}^{\dagger} \hat{a}+\frac{\omega_{b}}{2} \hat{\sigma}_{z}+g\left(\hat{a}-\hat{a}^{\dagger}\right)\left(\hat{\sigma}^{-}-\hat{\sigma}^{+}\right) \\
& \approx\left(\tilde{\omega}_{a} \hat{\tilde{a}}^{\dagger} \hat{\tilde{a}}-K_{a} \hat{\tilde{a}}^{\dagger 2} \hat{\tilde{a}}^{2}\right)+\frac{\tilde{\omega}_{b}}{2} \hat{\tilde{\sigma}}_{z}-\chi \hat{\tilde{a}}^{\dagger} \hat{\tilde{a}} \hat{\tilde{\sigma}}_{z} .
\end{aligned}
$$

However, the nonlinear coupling $\chi$ is only a perturbation to the underlying linear coupling $g$, which creates the dressed normal modes $\{\hat{\tilde{a}}, \hat{\tilde{b}}\}$ from a linear combination of the uncoupled bare modes $\{\hat{a}, \hat{b}\}$ [10]. This linear mixing causes detrimental effects like Purcell decay [11] in the atomic mode and induced self-Kerr coefficient $K_{a}$ in the photonic mode [12-19]. These effects are usually suppressed by keeping $\chi / 2 \pi \sim 10^{1} \mathrm{MHz}$. Furthermore, the high detuning $\Delta$ requirement leads to frequency crowding [20] and difficulty in scaling [21].

Recently, a number of devices [4,22-28] have been used as couplers to facilitate a purely nonlinear coupling between bare modes,

$$
\begin{aligned}
H & =\left(\omega_{a} \hat{a}^{\dagger} \hat{a}-K_{a} \hat{a}^{\dagger 2} \hat{a}^{2}\right)+\left(\omega_{b} \hat{b}^{\dagger} \hat{b}-K_{b} \hat{b}^{\dagger 2} \hat{b}^{2}\right)+\chi \hat{a}^{\dagger} \hat{a} \hat{b}^{\dagger} \hat{b} \\
& \approx \frac{\omega_{a}}{2} \hat{\sigma}_{z, a}+\frac{\omega_{b}}{2} \hat{\sigma}_{z, b}+\chi \hat{\sigma}_{z, a} \hat{\sigma}_{z, b},
\end{aligned}
$$

where the modes $\{\hat{a}, \hat{b}\}$ refer to distinct superconducting $L C$ oscillators [29]. The resulting nonlinear coupling of linearly decoupled [30] qubits is free from Purcell decay and requires no qubit frequency detuning. However, 
existing purely nonlinear couplers are limited to $\chi \leq K_{a, b}$ [26]. Couplers like the $C$-shunt superconducting quantum interference device (SQUID) [22-24] and the Josephson ring modulator (JRM) [26,27] induce significant self-Kerr coefficient in both modes $\{\hat{a}, \hat{b}\}$, which limits their applications to only $\chi \hat{\sigma}_{z} \hat{\sigma}_{z}$ qubit-qubit coupling [24,27]. Furthermore, since transmon qubits have limited self-Kerr coefficient $K_{a, b} / 2 \pi \sim 10^{2} \mathrm{MHz}$ [31], these qubit-qubit couplers have limited $\chi / 2 \pi \sim 10^{2} \mathrm{MHz}$ as well. See Supplemental Material [32] for clarifications of photon and qubit mode definitions.

In this Letter, we propose a scheme to achieve ultrastrong purely nonlinear coupling, which we define, in analogy with ultrastrong linear coupling, to be $\chi \sim 2 \pi \times 1 \mathrm{GHz} \gtrsim$ $0.1 \omega_{a, b}$ without linear coupling $(g=0)$. In addition, the scheme can cancel the self-Kerr coefficient $K_{a, b}$ such that the $\chi$ couples any combination of light and matter modesin stark contrast with the light-matter only coupling in the dispersive regime and matter-matter only coupling by the $C$-shunt SQUID or JRM. The scheme can thus enable the first light-light purely nonlinear coupling $\left(\chi \hat{a}^{\dagger} \hat{a} \hat{b}^{\dagger} \hat{b}\right)$, which is ideal for applications like high fidelity single microwave photon detection [8]. The 2 orders of magnitude improvement for light-matter coupling and 1 order of magnitude improvement for matter-matter coupling could enable ultrafast qubit readout [4] and two-qubit gates [5,6], Ising interaction dominated quantum annealing architectures [26], as well as self-Kerr free bosonic qubit control $[9,17,41,42]$.

The quarton.-The proposed purely nonlinear couplers are based on a superconducting qubit dubbed the "quarton" [43]. The quarton was recently demonstrated as a flux qubit with high anharmonicity and long coherence times [43]; devices with similar quartic potentials were proposed previously as a highly anharmonic phase qubit with efficient readout [44] and demonstrated as part of a superinductor [45].

We introduce the quarton here by first categorizing superconducting circuit elements by their nonlinearities, which are usually derived from the cosine potential of the Josephson junction (JJ) [46],

$U_{\mathrm{JJ}}(\phi)=-E_{J} \cos \phi \approx \frac{E_{J}}{2} \phi^{2}-\frac{E_{J}}{24} \phi^{4} \approx \frac{\phi_{0}^{2}}{2 L_{J}} \phi^{2}-K \phi^{4}$,

where $E_{J}$ is the Josephson energy. Physically, JJs with superconducting phase $\phi$ exhibit both a positive linear inductance $L_{J}$ given by the quadratic $\phi^{2}$ component of the potential and a negative nonlinear inductance given by the quartic $\phi^{4}$ (and higher-order) component of the potential. For the remainder of the Letter, we assume $\phi \ll 1$ and keep up to the quartic $\phi^{4}$ term, which is characterized by the nonlinear Kerr coefficient $K$.
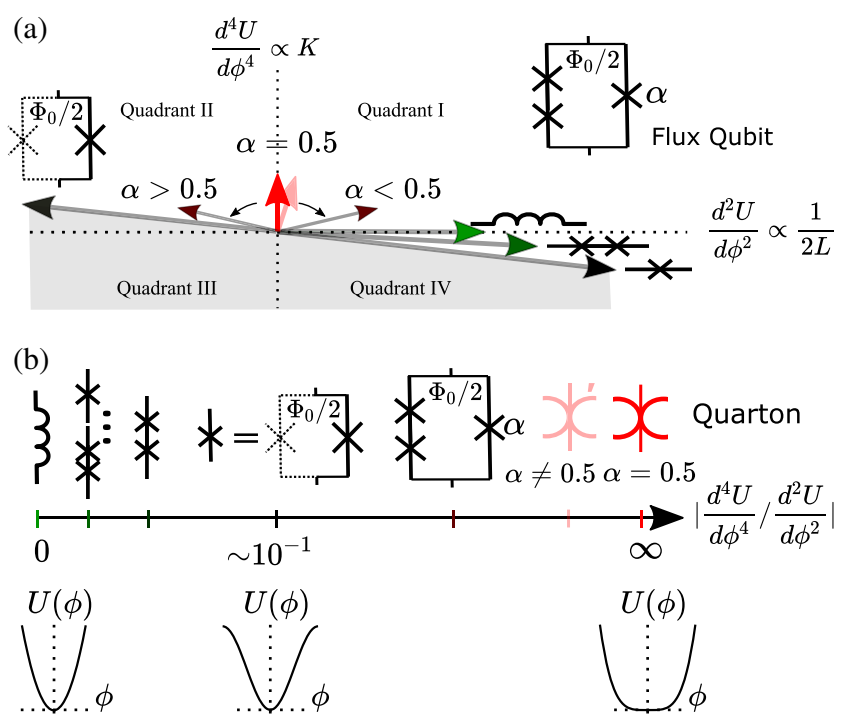

FIG. 1. The quarton as a purely nonlinear element. (a) Schematic plot of the nonlinear $(K)$ vs linear $(1 / 2 L)$ landscape of inductive superconducting elements with centrosymmetric potentials $(U)$. The flux qubit has a negative and a positive inductance branch with strength dependent on ratio $\alpha$. The quarton (red) is a special flux qubit with $\alpha=0.5$ that has no linear potential. The gray region is energetically unstable (see Supplemental Material [32]). (b) Schematic line scale of the relative nonlinearity of the elements in (a). The quarton (red spider symbol) is at infinity. The respective potentials $U(\phi)$ 's are plotted below. The tilted quarton (light red spider symbol with apostrophe) is a quarton with small linear inductive potential.

We repeat this for a wide range of inductive superconducting elements, which leads to a schematic plot of their nonlinear $\left(d^{4} U / d \phi^{4}\right)$ vs linear $\left(d^{2} U / d \phi^{2}\right)$ energy coefficient in Fig. 1(a) [47]. For ease of comparison, the slope $\left|\left(d^{4} U / d \phi^{4}\right) /\left(d^{2} U / d \phi^{2}\right)\right|$ is plotted in Fig. 1(b) with the potential diagrams $U(\phi)$ illustrated at the bottom. Following Eq. (3), we place the JJ as a vector in quadrant IV of Fig. 1(a) with a length proportional to $E_{J}$. However, the slope or direction of the JJ vector that characterizes its relative nonlinearity is invariant with $E_{J}$. We can thus think of the linear-nonlinear plane [Fig. 1(a)] as a two-dimensional vector space, with different circuit elements as vectors having $E_{J}$-dependent length but unique directionality.

We consider three techniques that change the relative nonlinearity: (i) add more JJs in series to decrease the relative nonlinearity $[48,49]$, (ii) thread half a flux quantum $\left(\Phi_{0} / 2\right)$ of external magnetic flux through a loop of elements, and (iii) connect inductive elements in parallel to add their vectors on Fig. 1(a). For (i) with $n$ identical JJs in series (all with $E_{J} \gg E_{C}, E_{C}$ being capacitive energy [46]), the phase $\phi$ across the chain of JJs is divided evenly across each $\mathrm{JJ}(\phi \rightarrow \phi / n)$ [48]. By Eq. (3), this implies that: $(1 / L) \rightarrow(n / L)\left(1 / n^{2}\right), K \rightarrow n K\left(1 / n^{4}\right)$, so more JJs in series lowers $\left|\left(d^{4} U / d \phi^{4}\right) /\left(d^{2} U / d \phi^{2}\right)\right|$. In the limit 
$n \rightarrow \infty$, we get a superinductor [48], which is purely linear. We can also (ii) add a $\Phi_{0} / 2$ flux bias: for a multibranch element like a SQUID, the external flux acts on one JJ branch [50] and shifts its cosine potential $U_{\mathrm{JJ}}(\phi) \rightarrow$ $-U_{\mathrm{JJ}}(\phi)$ via $\phi \rightarrow \phi+\pi$. This flips one branch [50] JJ vector to the quadrant II of Fig. 1(a). We can further use (iii) to add vectors to produce devices such as flux qubits that live in the space between the flux-biased and unbiased SQUIDs and/or JJs. This is valid because flux qubits, in general, have two parallel branches with the same $\phi$, so the overall potential $U(\phi)$ is a sum of the two branch $U$ 's.

The top right corner of Fig. 1(a) shows a conventional flux qubit [43] with two identical JJs with $E_{J}$ in series in one branch, and a smaller area JJ with $\alpha E_{J}$ in the other branch. Without loss of generality [50], we choose the gauge such that the $\alpha E_{J}$ branch is flux biased [quadrant II of Fig. 1(a)] and the series JJ branch is unchanged [quadrant IV of Fig. 1(a)]. Because the two branches have different $\left|\left(d^{4} U / d \phi^{4}\right) /\left(d^{2} U / d \phi^{2}\right)\right|$ [Fig. 1(b)], the resulting flux qubit vector from the addition of the two branch vectors can have different directions depending on $\alpha$. Flux qubits with more $(n \geq 2)$ series JJs follow the same principle, with potential [51]

$$
U(\phi)=-n E_{J} \cos \left(\frac{\phi}{n}\right)-\alpha E_{J} \cos (\phi-\pi) .
$$

The quarton is the special flux qubit with $\alpha=0.5(=1 / n$, in general), for which the negative inductance from the quadrant II vector exactly cancels the positive inductance from the quadrant IV vector, while the stronger positive $K$ of the quadrant II vector survives the addition. The quarton is named after the resulting leading-order positive quartic $\phi^{4}$ potential and zero $\phi^{2}$ potential. Physically, the linearized current flow in the two branches destructively interfere. In Fig. 1(b), the quarton (spider symbol [53]) defines the infinity end of the $\left|\left(d^{4} U / d \phi^{4}\right) /\left(d^{2} U / d \phi^{2}\right)\right|$ scale opposite to the linear inductor that defines the zero point. Graphically, the potential diagrams below Fig. 1(b) show a completely anharmonic quartic potential for the quarton. See Supplemental Material [32] for a discussion on a nonlinear optics analogy and the energetically unstable gray region in Fig. 1(a).

In practice, JJ-based inductive elements like the quarton have accompanying junction capacitances that can cause linear capacitive coupling. To mitigate this, we use a slightly linear quarton, dubbed the "tilted quarton" for its position in Fig. 1(a) (light red). The tilted quarton has some small linear inductive potential, which can cancel the coupling effects of an equally small amount of accompanying linear capacitance [22,24]. To distinguish it from the quarton, we give the tilted quarton a lightly shaded spider symbol with an extra apostrophe [Fig. 1(b)].

Self-Kerr coefficient cancellation. - Consider the canonical circuit of two transmon qubits (labeled $a$ and $b$ ) coupled via a quarton, shown in Fig. 2(a). We can construct an exact
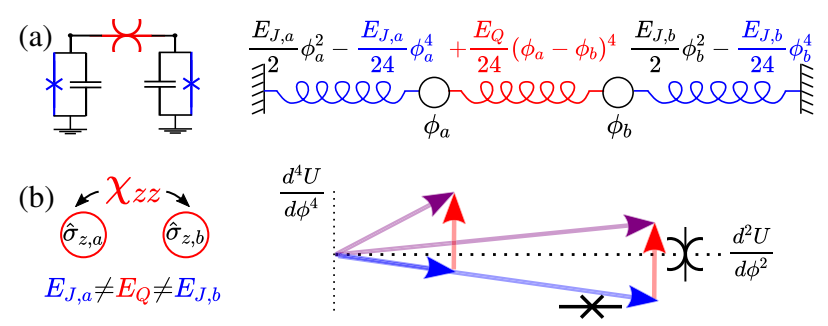

(c)
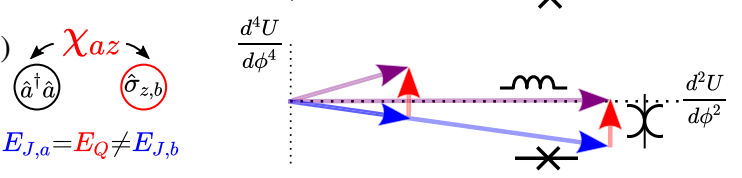

$E_{J, a}=E_{Q} \neq E_{J, b}$
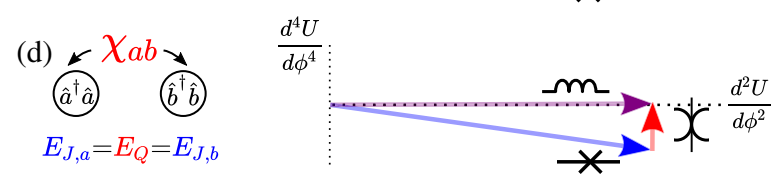

FIG. 2. Quarton-mediated purely nonlinear light and/or matter interactions. (a) Canonical circuit of quarton (red) nonlinearly coupling two transmons (blue) indexed $a, b$, and its spring-mass analog with Josephson energies as nonlinear spring coefficients $E_{J, a}, E_{Q}, E_{J, b}$. Strong nonlinear coupling between (b) mattermatter modes when $\left(E_{J, a} \neq E_{Q} \neq E_{J, b}\right)$ quarton induces positive qubit nonlinearities, (c) light-matter modes when $\left(E_{J, a}=E_{Q} \neq\right.$ $\left.E_{J, b}\right)$ quarton cancels qubit $a$ 's self-Kerr nonlinearity, (d) lightlight modes when $\left(E_{J, a}=E_{Q}=E_{J, b}\right)$ quarton cancels both qubits' self-Kerr nonlinearity. Photon annihilation operators, $\hat{a}$, $\hat{b}$; qubit operators, $\hat{\sigma}_{z, a b}$.

spring-mass analogy for the system wherein $\phi$ and $E_{J}$ are analogs of position and spring constant, respectively. $\left(E_{Q} \equiv E_{J}\left(n^{2}-1 / n^{3}\right)\right.$ is the effective Josephson energy of the quarton, see Supplemental Material [32] for details.) Note that because there is no linear coupling potential of the form $\left(E_{Q} / 2\right)\left(\phi_{a}-\phi_{b}\right)^{2}$ in the red quarton spring, the quarton naturally facilitates purely nonlinear coupling without linear coupling.

Remarkably, by simply adjusting the relative magnitudes of the qubit spring constants $\left(E_{J, a}, E_{J, b}\right)$ to the coupling quarton spring constant $\left(E_{Q}\right)$, we can access nonlinear coupling between all three combinations of light and matter modes. As shown in Figs. 2(b)-2(d), these combinations are qubit-qubit $\left(\chi_{z z} \hat{\sigma}_{z} \hat{\sigma}_{z}\right)$, qubit-photon $\left(\chi_{a z} \hat{a}^{\dagger} \hat{a} \hat{\sigma}_{z}\right)$, and photonphoton $\left(\chi_{a b} \hat{a}^{\dagger} \hat{a} \hat{b}^{\dagger} \hat{b}\right)$ cross-Kerr coupling, respectively. In particular, Fig. 2(d) represents the first system that exhibits cross-Kerr coupling without self-Kerr nonlinearity or photon-photon purely nonlinear coupling. This is in stark contrast with previous purely nonlinear coupling schemes $[22,26,30]$ that leave modes with nonzero selfKerr coefficient [25].

The quarton's purely nonlinear coupling potential $\left(E_{Q} / 24\right)\left(\hat{\phi}_{a}-\hat{\phi}_{b}\right)^{4}$ can be expanded into

$\frac{E_{Q}}{24}\left(\hat{\phi}_{a}-\hat{\phi}_{b}\right)^{4}=\frac{E_{Q}}{24}\left[\hat{\phi}_{a}^{4}+\hat{\phi}_{b}^{4}+6 \hat{\phi}_{a}^{2} \hat{\phi}_{b}^{2}-4\left(\hat{\phi}_{a}^{3} \hat{\phi}_{b}+\hat{\phi}_{a} \hat{\phi}_{b}^{3}\right)\right]$. 
After quantizing $\hat{\phi}_{a}=\phi_{\mathrm{ZPF}, a}\left(\hat{a}+\hat{a}^{\dagger}\right), \hat{\phi}_{b}=\phi_{\mathrm{ZPF}, b}\left(\hat{b}+\hat{b}^{\dagger}\right)$, the $\hat{\phi}_{a}^{2} \hat{\phi}_{b}^{2}$ term leads to the important cross-Kerr $\left(\hat{a}^{\dagger} \hat{a} \hat{b}^{\dagger} \hat{b}\right)$-type nonlinear coupling and the $\hat{\phi}_{a, b}^{3} \hat{\phi}_{b, a}$ terms induce other four wave mixing nonlinear effects, including correlated photon hopping [24], third harmonic generation [54], parametric amplification, and squeezing [55]. Importantly, the positive, noncoupling terms $+\left(E_{Q} / 24\right) \hat{\phi}_{a, b}^{4}$ can be grouped with the qubits' negative nonlinear potentials $-\left(E_{J, a b} / 24\right) \hat{\phi}_{a, b}^{4}$ to produce effective qubit nonlinear potentials of $\left[\left(E_{Q}-E_{J, a b}\right) / 24\right] \hat{\phi}_{a, b}^{4}$. This can be intuitively represented on the linear-nonlinear diagram for each case. As shown in Figs. 2(b)-2(d), when $E_{J a, b} \neq E_{Q}$, the vector sum (purple) of the quarton vector (red) and the $\mathrm{JJ}$ vector (blue) is nonzero in the nonlinear axis; this represents residual resonator self-Kerr nonlinearity in a qubit mode. In contrast, when $E_{J a, b}=E_{Q}$, the quarton's induced positive self-Kerr cancels the JJ's intrinsic negative self-Kerr coefficient and the resulting sum is zero in the vertical nonlinear axis; this represents a linear resonator photon mode. We emphasize that in situ tuning of $E_{J, a}$ and $E_{J, b}$ is possible with flux-tunable transmons [31]. Therefore, results here enable flexible superconducting architectures with modes that can be tuned in situ to behave either as qubits or resonators, potentially enabling work like Ref. [56].

Ultrastrong $\chi$.-We contrast potential realizations of the canonical circuit in Fig. 2(a) with two state-of-the-art nonlinear couplers: the $C$-shunt SQUID [22-25], which cancels inductive and capacitive linear coupling within the rotating wave approximation (RWA), and the Josephson ring modulator [26,27], which cancels all linear coupling as well as asymmetric nonlinear coupling $\left(\phi_{a, b}^{3} \phi_{b, a}\right)$ terms by symmetry. The two qubits $a$ and $b$ to be coupled are typical transmons and properties are calculated both analytically and numerically using QuCAT [57]. See Supplemental Material [32] for related derivations and calculations.

Analogous to the $C$-shunt SQUID, we use the tilted quarton [Fig. 3(a)] to cancel (up to RWA) the linear coupling due to intrinsic junction capacitances $C_{J}$. Unlike the $C$-shunt SQUID [Fig. 3(b)], which needs a large, variable shunt capacitance $C_{J}$ to cancel the SQUID inductance, the tilted quarton has intentionally added inductance to the quarton to cancel a small, fixed $C_{J}$. Henceforth, we use $E_{Q}$ to denote both the quarton's and the corresponding $C$-shunt SQUID and JRM's Josephson energy. In Fig. 3(c), we show that, for large $E_{Q}$, quarton-enabled nonlinear coupling strength $\chi$ (for $\hat{a}^{\dagger} \hat{a} \hat{b}^{\dagger} \hat{b}$ in $\phi_{a}^{2} \phi_{b}^{2}$ ) can be an order of magnitude (1 GHz vs $100 \mathrm{MHz}$ ) higher than the $C$-shunt SQUID, which limits $\chi$ to much less than the anharmonicities $E_{c, a}$ and $E_{c, b}$ of the transmons. This is because all existing couplers have linear inductive potentials that increase $E_{J, a b}$ of the qubits to an effective $E_{Q}+E_{J, a b}$; or in the springmass analogy [Fig. 2(a)], the $a$ and $b$ masses oscillate
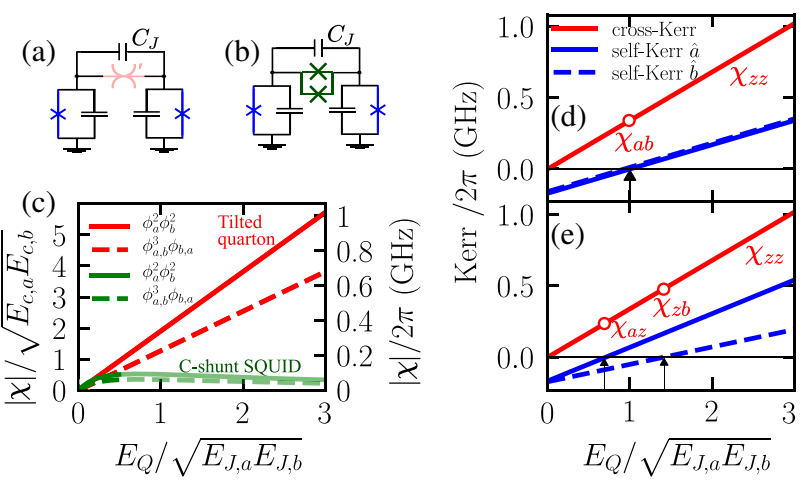

FIG. 3. Purely nonlinear coupling (both $\phi_{a}^{2} \phi_{b}^{2}$ and $\phi_{a, b}^{3} \phi_{b, a}$ ) between qubits with tunable frequencies less than $9 \mathrm{GHz}$, mediated by (a) tilted quarton (red) with $C_{J}=5 \mathrm{fF}$ vs (b) $C$ shunt SQUID [22] (green). (c) Nonlinear coupling $\chi$ (for $\hat{a}^{\dagger} \hat{a} \hat{b}^{\dagger} \hat{b}$ in $\phi_{a}^{2} \phi_{b}^{2}$ and similar for $\phi_{a, b}^{3} \phi_{b, a}$ ) scales linearly with $E_{Q}$ for tilted quarton, allowing for order of magnitude improvement over $C$ shunt SQUID at large $E_{Q}$. $C$-shunt SQUID's linear coupling cancellation relies on RWA, which is invalid for large $E_{Q}$ (light green). For tilted quarton, (d) simultaneous self-Kerr $\left(\hat{a}^{\dagger 2} \hat{a}^{2}, \hat{b}^{\dagger 2} \hat{b}^{2}\right)$ cancellation is possible with $E_{J, a}=E_{J, b}$, which is also used in (c). (e) Same qubits flux tuned to $E_{J, a} \neq E_{J, b}$ leads to self-Kerr cancellation of only one mode at a time.

in a stiffer $\left[\left(E_{J, a b}+E_{Q}\right) / 2\right] \phi_{a, b}^{2}$ spring potential if $\left(E_{Q} / 2\right)\left(\phi_{a}-\phi_{b}\right)^{2}$ exists. The stiffer spring reduces oscillation amplitude, or the zero point fluctuation $\phi_{\mathrm{ZPF}, a b}^{4}=\left(2 E_{C, a b} / E_{J, a b}\right) \rightarrow\left[2 E_{C, a b} /\left(E_{Q}+E_{J, a b}\right)\right]$ quantum mechanically, which directly reduces the coupling $[22,26]$

$$
\chi(\text { nonquarton }) \propto \frac{\sqrt{E_{c, a} E_{c, b}} E_{Q}}{\sqrt{E_{Q}+E_{J, a}} \sqrt{E_{Q}+E_{J, b}}},
$$

which has $\lim _{E_{Q} \gg E_{J, a b}} \chi \leq \sqrt{E_{c, a} E_{c, b}}$. Using the quarton instead, we can avoid the detrimental linear inductance induced $E_{J, a b} \rightarrow E_{Q}+E_{J, a b}$ and achieve

$$
\chi(\text { quarton }) \propto \sqrt{E_{c, a} E_{c, b}} \frac{E_{Q}}{\sqrt{E_{J, a}} \sqrt{E_{J, b}}},
$$

which offers approximately linear scaling $\chi \propto E_{Q}$. See Supplemental Material [32] for a detailed derivation of Eqs. (6) and (7) and the limit to Eq. (7).

In Figs. 3(d) and 3(e), we examine the self-Kerr $\hat{a}^{\dagger 2} \hat{a}^{2}$, $\hat{b}^{\dagger 2} \hat{b}^{2}$ (blue) and cross-Kerr $\hat{a}^{\dagger} \hat{a} \hat{b}^{\dagger} \hat{b}$ (red) magnitudes for the qubits coupled by tilted quarton and show that all three regimes in Figs. 2(b)-2(d) can be reached (whereas $C$-shunt SQUID can only have $\chi_{z z}$ ). For two identical qubits that guarantees $E_{J, a}=E_{J, b}$ [Fig. 3(d)], we have that the quarton cancels both qubit self-Kerr coefficients when $E_{Q}=$ $E_{J, a}=E_{J, b}$ (black arrow). At that point, there still exists 
(a)

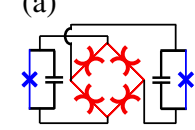

(b)

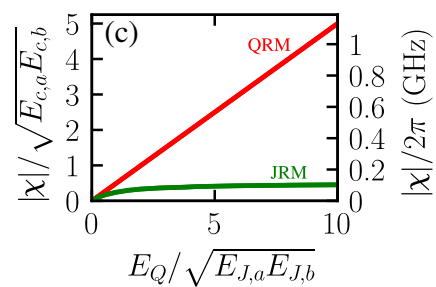

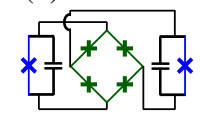

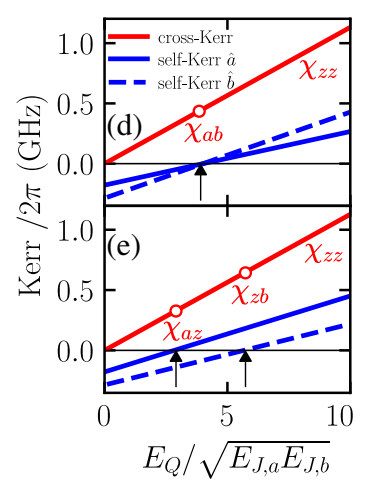

FIG. 4. Purely cross-Kerr $\left(\phi_{a}^{2} \phi_{b}^{2}\right)$-type nonlinear coupling between qubits with tunable frequencies less than $7 \mathrm{GHz}$, mediated by (a) QRM (red) vs (b) JRM [26] (green). (c) Cross-Kerr strength $\chi$ (for $\hat{a}^{\dagger} \hat{a} \hat{b}^{\dagger} \hat{b}$ in $\phi_{a}^{2} \phi_{b}^{2}$ ) scales linearly with $E_{Q}$ for QRM, allowing for order of magnitude improvement over JRM at large $E_{Q}$. For QRM, (d) simultaneous self-Kerr $\left(\hat{a}^{\dagger 2} \hat{a}^{2}, \hat{b}^{\dagger 2} \hat{b}^{2}\right)$ cancellation is possible with $E_{J, a}=E_{J, b}$, which is also used in (c). (e) Same qubits flux tuned to $E_{J, a} \neq E_{J, b}$ leads to self-Kerr cancellation of only one mode at a time.

a relatively large $\chi_{a b}$, enabling strong single photon-photon interactions. With the same qubits flux tuned to different $E_{J, a} \neq E_{J, b}$ [Fig. 3(e)], we can have qubit-photon nonlinear couplings $\chi_{a z}, \chi_{z b}$ at $E_{Q}=E_{J, a} \neq E_{J, b}$ and $E_{Q}=$ $E_{J, b} \neq E_{J, a}$ (black arrows), respectively. In general, when $E_{Q} \neq E_{J, a} \neq E_{J, b}$, we have qubit-qubit $\chi_{z z}$ nonlinear coupling.

We draw a similar comparison in Fig. 4 by constructing a quarton ring modulator (QRM) in Fig. 4(a) with the same symmetry as the JRM. The symmetry guarantees that only cross-Kerr-type $\left(\phi_{a}^{2} \phi_{b}^{2}\right)$ nonlinear coupling terms exist, and junction capacitances $C_{J}$ do not cause any linear coupling [58]. In Fig. 4(c), we find a similar giant $(>1 \mathrm{GHz}) \chi$ reachable via the QRM, which is an order of magnitude improvement over the JRM. Although the JRM can operate with higher $E_{Q}$ compared to the $C$-shunt SQUID [22,26], its linear inductive potential still limits its $\chi \leq \sqrt{E_{c, a} E_{c, b}}$ by Eq. (6). Unlike the JRM, self-Kerr cancellation by the QRM [Figs. 4(d) and 4(e)] can lead to purely cross-Kerrtype coupling between any combination of light and matter modes.

Discussion.-The proposed circuits are experimentally feasible (see Supplemental Material [32] for details) and lead to important applications such as ultrafast readout and two-qubit gates. Qubit readout uses resonator-qubit crossKerr coupling $\chi \hat{a}^{\dagger} \hat{a} \hat{\sigma}_{z}$, which leads to readout times that scale roughly as $1 / \chi$ [33]. Similarly, two-qubit gates such as the resonator-induced phase gate $[5,6]$ uses cross-Kerr between two qubits and a shared resonator $\chi \hat{a}^{\dagger} \hat{a}\left(\hat{\sigma}_{z, 1}+\hat{\sigma}_{z, 2}\right)$, which leads to gate times that scale as $1 / \chi^{2}$. These readout and gate procedures are all currently implemented with the small dispersive $\chi$ coupling; if instead, our proposed ultrastrong quarton-based $\chi$ is used, the readout and gate times could be reduced by orders of magnitude.

Conclusion.-We showed that quarton-based couplers (tilted quarton and QRM) can facilitate ultrastrong $(\sim 1 \mathrm{GHz})$ purely nonlinear coupling between any combination of qubit and photon modes: qubit-qubit, qubitphoton, and, uniquely, photon-photon. Unlike previous nonlinear couplers, the ability to couple to photon modes enables applications in ultrafast readout, gates, bosonic qubit control, and single photon detection.

This work was funded in part by the MIT Center for Quantum Engineering via support from the Laboratory for Physical Sciences under Contract No. H98230-19-C-0292. Y. Y. acknowledges financial support from the MIT EECS Jin Au Kong Fellowship. G. C. acknowledges support from the Harvard Graduate School of Arts and Sciences Prize Fellowship. The authors thank Arne L. Grimsmo, William D. Oliver, and Terry P. Orlando for fruitful discussions and insightful comments.

*To whom all correspondence should be addressed. kpobrien@mit.edu

[1] M. H. Devoret and R. J. Schoelkopf, Science 339, 1169 (2013).

[2] F. Arute, K. Arya, R. Babbush, D. Bacon, J. C. Bardin, R. Barends, R. Biswas, S. Boixo, F. G. Brandao, D. A. Buell et al., Nature (London) 574, 505 (2019).

[3] T. Niemczyk, F. Deppe, H. Huebl, E. Menzel, F. Hocke, M. Schwarz, J. Garcia-Ripoll, D. Zueco, T. Hümmer, E. Solano et al., Nat. Phys. 6, 772 (2010).

[4] R. Dassonneville, T. Ramos, V. Milchakov, L. Planat, É. Dumur, F. Foroughi, J. Puertas, S. Leger, K. Bharadwaj, J. Delaforce et al., Phys. Rev. X 10, 011045 (2020).

[5] S. Puri and A. Blais, Phys. Rev. Lett. 116, 180501 (2016).

[6] H. Paik, A. Mezzacapo, M. Sandberg, D. T. McClure, B. Abdo, A. D. Córcoles, O. Dial, D. F. Bogorin, B. L. T. Plourde, M. Steffen et al., Phys. Rev. Lett. 117, 250502 (2016).

[7] S. Kono, K. Koshino, Y. Tabuchi, A. Noguchi, and Y. Nakamura, Nat. Phys. 14, 546 (2018).

[8] A. L. Grimsmo, B. Royer, J. M. Kreikebaum, Y. Ye, K. O'Brien, I. Siddiqi, and A. Blais, Phys. Rev. Applied 15, 034074 (2021).

[9] S. Krastanov, V. V. Albert, C. Shen, C.-L. Zou, R. W. Heeres, B. Vlastakis, R. J. Schoelkopf, and L. Jiang, Phys. Rev. A 92, 040303(R) (2015).

[10] A. Blais, R.-S. Huang, A. Wallraff, S. M. Girvin, and R. J. Schoelkopf, Phys. Rev. A 69, 062320 (2004).

[11] N. Didier, J. Bourassa, and A. Blais, Phys. Rev. Lett. 115, 203601 (2015).

[12] G. Kirchmair, B. Vlastakis, Z. Leghtas, S. E. Nigg, H. Paik, E. Ginossar, M. Mirrahimi, L. Frunzio, S. M. Girvin, and R. J. Schoelkopf, Nature (London) 495, 205 (2013).

[13] T. Peronnin, D. Marković, Q. Ficheux, and B. Huard, Phys. Rev. Lett. 124, 180502 (2020). 
[14] A. L. Grimsmo, J. Combes, and B. Q. Baragiola, Phys. Rev. X 10, 011058 (2020).

[15] Z. Leghtas, G. Kirchmair, B. Vlastakis, M. H. Devoret, R. J. Schoelkopf, and M. Mirrahimi, Phys. Rev. A 87, 042315 (2013).

[16] S. Rosenblum, Y. Y. Gao, P. Reinhold, C. Wang, C. J. Axline, L. Frunzio, S. M. Girvin, L. Jiang, M. Mirrahimi, M. H. Devoret et al., Nat. Commun. 9, 1 (2018).

[17] J. Joo and E. Ginossar, Sci. Rep. 6, 1 (2016).

[18] B. Vlastakis, G. Kirchmair, Z. Leghtas, S. E. Nigg, L. Frunzio, S. M. Girvin, M. Mirrahimi, M. H. Devoret, and R. J. Schoelkopf, Science 342, 607 (2013).

[19] S. Puri, S. Boutin, and A. Blais, npj Quantum Inf. 3, 18 (2017).

[20] R. Schutjens, F. A. Dagga, D. J. Egger, and F. K. Wilhelm, Phys. Rev. A 88, 052330 (2013).

[21] C. Berke, E. Varvelis, S. Trebst, A. Altland, and D. P. DiVincenzo, arXiv:2012.05923.

[22] L. Neumeier, M. Leib, and M. J. Hartmann, Phys. Rev. Lett. 111, 063601 (2013).

[23] J. Jin, D. Rossini, R. Fazio, M. Leib, and M. J. Hartmann, Phys. Rev. Lett. 110, 163605 (2013).

[24] M. Kounalakis, C. Dickel, A. Bruno, N. K. Langford, and G. A. Steele, npj Quantum Inf. 4, 38 (2018).

[25] M. C. Collodo, A. Potočnik, S. Gasparinetti, J.-C. Besse, M. Pechal, M. Sameti, M. J. Hartmann, A. Wallraff, and C. Eichler, Phys. Rev. Lett. 122, 183601 (2019).

[26] M. Leib, P. Zoller, and W. Lechner, Quantum Sci. Technol. 1, 015008 (2016).

[27] T. Roy, S. Kundu, M. Chand, S. Hazra, N. Nehra, R. Cosmic, A. Ranadive, M. P. Patankar, K. Damle, and R. Vijay, Phys. Rev. Applied 7, 054025 (2017).

[28] E. T. Holland, B. Vlastakis, R. W. Heeres, M. J. Reagor, U. Vool, Z. Leghtas, L. Frunzio, G. Kirchmair, M. H. Devoret, M. Mirrahimi, and R. J. Schoelkopf, Phys. Rev. Lett. 115, 180501 (2015).

[29] M. Devoret, B. Huard, R. Schoelkopf, and L. F. Cugliandolo, Quantum Machines: Measurement and Control of Engineered Quantum Systems (2011), Vol. 96, https://oxford.universitypressscholarship.com/view/10.1093/ acprof:oso/9780199681181.001.0001/acprof-9780199681181.

[30] M. Menotti, B. Morrison, K. Tan, Z. Vernon, J. E. Sipe, and M. Liscidini, Phys. Rev. Lett. 122, 013904 (2019).

[31] J. Koch, T. M. Yu, J. Gambetta, A. A. Houck, D. I. schuster, J. Majer, A. Blais, M. H. Devoret, S. M. Girvin, and R. J. Schoelkopf, Phys. Rev. A 76, 042319 (2007).

[32] See Supplemental Material at http://link.aps.org/ supplemental/10.1103/PhysRevLett.127.050502 for more discussion, which includes Refs. [33-40].

[33] A. Blais, A. L. Grimsmo, S. M. Girvin, and A. Wallraff, Rev. Mod. Phys. 93, 025005 (2021).

[34] T. Roy, S. Hazra, S. Kundu, M. Chand, M. P. Patankar, and R. Vijay, Phys. Rev. Applied 14, 014072 (2020).

[35] W. Zhang, W. Huang, M. E. Gershenson, and M. T. Bell, Phys. Rev. Applied 8, 051001(R) (2017).
[36] D. L. Andrews, Photonics, Volume 1: Fundamentals of Photonics and Physics (John Wiley \& Sons, New Jersey, 2015).

[37] V. Shnyrkov, A. Soroka, and S. Melnyk, Low Temp. Phys. 34, 610 (2008).

[38] L. V. Abdurakhimov, I. Mahboob, H. Toida, K. Kakuyanagi, and S. Saito, Appl. Phys. Lett. 115, 262601 (2019).

[39] C. K. Andersen and A. Blais, New J. Phys. 19, 023022 (2017).

[40] J. Kreikebaum, K. O’Brien, A. Morvan, and I. Siddiqi, Supercond. Sci. Technol. 33, 06LT02 (2020).

[41] M. Elliott, J. Joo, and E. Ginossar, New J. Phys. 20, 023037 (2018).

[42] J. Joo, C.-W. Lee, S. Kono, and J. Kim, Sci. Rep. 9, 16592 (2019).

[43] F. Yan, Y. Sung, P. Krantz, A. Kamal, D. K. Kim, J. L. Yoder, T. P. Orlando, S. Gustavsson, and W. D. Oliver, arXiv:2006.04130.

[44] A. B. Zorin and F. Chiarello, Phys. Rev. B 80, 214535 (2009).

[45] M. T. Bell, I. A. Sadovskyy, L. B. Ioffe, A. Y. Kitaev, and M. E. Gershenson, Phys. Rev. Lett. 109, 137003 (2012).

[46] M. Tinkham, Introduction to Superconductivity (Courier Corporation, Mineola, 2004).

[47] Note that we include only symmetric potentials and neglect higher-order nonlinearities for simplicity.

[48] N. A. Masluk, I. M. Pop, A. Kamal, Z. K. Minev, and M. H. Devoret, Phys. Rev. Lett. 109, 137002 (2012).

[49] V. V. Sivak, S. Shankar, G. Liu, J. Aumentado, and M. H. Devoret, Phys. Rev. Applied 13, 024014 (2020).

[50] X. You, J. A. Sauls, and J. Koch, Phys. Rev. B 99, 174512 (2019).

[51] This approximation is accurate in the limit that $E_{J} \gg E_{C}$ for each JJ and when $C_{g} \ll C_{J} / n^{2}$ where $C_{g}$ is each junction's capacitance to ground [45], which is straightforward to achieve in experiments [52].

[52] N. E. Frattini, V. V. Sivak, A. Lingenfelter, S. Shankar, and M. H. Devoret, Phys. Rev. Applied 10, 054020 (2018).

[53] V. E. Manucharyan, E. Boaknin, M. Metcalfe, R. Vijay, I. Siddiqi, and M. Devoret, Phys. Rev. B 76, 014524 (2007).

[54] R. W. Boyd, Nonlinear Optics (Academic Press, New York, 2019).

[55] C. Macklin, K. O’Brien, D. Hover, M. E. Schwartz, V. Bolkhovsky, X. Zhang, W. Oliver, and I. Siddiqi, Science 350, 307 (2015).

[56] A. Grimm, N. E. Frattini, S. Puri, S. O. Mundhada, S. Touzard, M. Mirrahimi, S. M. Girvin, S. Shankar, and M. H. Devoret, Nature (London) 584, 205 (2020).

[57] M. F. Gely and G. A. Steele, New J. Phys. 22, 013025 (2020).

[58] Note that, with large shunt $C_{J}$, the QRM can support a quadruple quarton mode in addition to modes $a$ and $b$. This enables a quarton alternative to the transmon based trimon [27], with cross-Kerr-coupled quarton-photon-photon or quartontransmon-photon or quarton-transmon-transmon modes. 